\title{
Imaging characteristics of metallic interbody spacers: in vitro score evaluation of susceptibility artifacts considering different MRI sequences
}

\author{
T. Ernstberger ${ }^{1,2}$, G. Heidrich ${ }^{3}$, G. Buchhorn ${ }^{4}$
}

${ }^{1}$ Clinic for Spinal Surgery, Klinikum Bad Bramstedt, Bad Bramstedt, Germany;

${ }^{2}$ Department of Orthopedic Surgery, University of Goettingen, Goettingen, Germany;

${ }^{3}$ Department of Diagnostic Radiology, University of Goettingen, Goettingen, Germany;

${ }^{4}$ Biomaterials Laboratory/Department of Orthopedic Surgery, University of Goettingenm, Goettingen, Germany.

Email: ernstberger@klinikumbb.de, ternstberger@med.uni-goettingen.de

Received 2 December 2009; revised 10 December 2009; accepted 14 December 2009.

\begin{abstract}
Aim: Intervertebral spacers for anterior spine fusion are made of different materials, such as titanium, carbon or cobalt-chrome, which can affect the postfusion MRI scans. Implant-related susceptibility artifacts can decrease the quality of MRI scans, thwarting proper evaluation. This cadaver study aimed to demonstrate the extent that implant-related MRI artifacting affects the post-fusion evaluation of intervertebral spacers. Methods: In a cadaveric porcine spine, we evaluated the post-implantation MRI scans of 2 metallic intervertebral spacers (TiAL6V4, CoCrMo) that differed in shape, material, surface qualities and implantation technique. A spacer made of human cortical bone was used as a control. The median sagittal MRI slice was divided into 12 regions of interest (ROI). Results: No significant differences were found on 15 different MRI sequences read independently by an interobserver-validated team of specialists $(P>0.05)$. Artifact-affected image quality was rated on a score of 0-1-2. A maximum score of 24 points $(100 \%)$ was possible. Turbo spin echo sequences produced the best scores for all spacers and the control. Only the control achieved a score of $100 \%$. The titanium and cobalt-chrome spacers scored $62.5 \%$ and $50 \%$, respectively. Conclusions: Our scoring system allowed us to create an implant-related ranking of MRI scan quality in reference to the control that was independent of artifact dimensions. Even with turbo spin echo sequences, the susceptibility artifacts produced by the metallic spacers showed a high degree of variability. Despite optimum sequencing, implant design and material are relevant factors in MRI artifacting.
\end{abstract}

Keywords: Intervertebral Spacers; Metallic Implant Materials; MRI; Susceptibility Artifacts

\section{INTRODUCTION}

In the preoperative diagnostics of spinal diseases, magnetic resonance imaging (MRI) is used as a standard procedure that can visualize disc pathologies and neurological changes of the spinal canal with high precision. When anterior spine fusion proves indicated, implantation of intervertebral spacers often represents the treatment of choice. The selection of implant design, implant material and implantation technique is dictated by the diagnostic findings. Intervertebral spacers made of various materials can be used as stand-alone cages for exclusively anterior fusion or in combination with dorsal instrumentation for dorsoventral fusion.

When postoperative complications arise secondary to vertebra fusion, MRI scans are frequently necessary to evaluate implant position and demonstrate any clinically relevant abnormalities and to direct further surgical decision-making [1]. Depending on the material, however, implant-related susceptibility artifacts can decrease the quality of MRI scans, thereby thwarting proper evaluation. Depending on the problem to be clarified, consideration must be given to whether the MRI sequence selected will ensure the most artifact-free visualization and enable proper evaluation of implant positioning and/or pathological processes like tumorous growth or infection.

Recent studies have shown that artifacting, particularly caused by metallic implants, can also be minimized through modification of routine MRI sequences $[2,3,4]$. This cadaveric porcine study was conducted to determine the extent to which implant-related MRI artifacting affects the evaluation of intervertebral spacers. A scoring system $(0-1-2)$ was developed to rank the artifacting produced by different intervertebral spacer designs compared with a human cortical bone control. Scans taken with 15 different MRI sequences were read independently by an interobserver-validated team of specialists who ranked image 


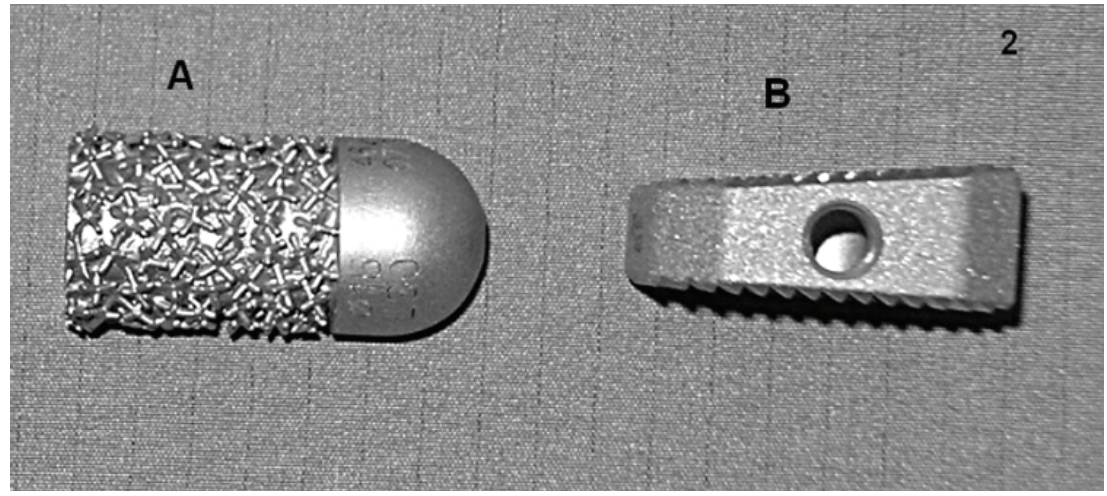

Figure 1. Intervertebrals disc spacers.

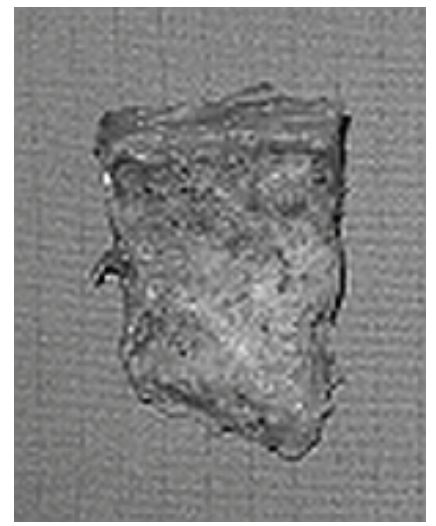

Figure 2. Human cortical bone.

quality of the implant, paying special attention to neighboring structures. The scores are presented in tables and possible implant-related factors discussed.

\section{MATERIAL AND METHODS}

In this study, we performed MRI on 2 implanted metallic intervertebral spacers (TiAl6V4, CoCrMo) (Figures 1(A) and $1(\mathrm{~B})$ ) that differed in shape, material, surface qualities and implantation technique. The spinal column of a domestic pig killed for commercial human consumption purchased from a slaughterhouse served as our experimental cadaveric model. The 2 spacers were implanted in the distal third of the thoracic spine and in the entire lumbar spine. Additionally, a piece of human cortical bone was implanted as a control (Figure 2).

\subsection{Spacers and Control}

The Intervertebral Body Spacer (IBS), manufactured by Peter Brehm GmbH, Chirurgie Mechanik, Weisendorf, Germany, is made of a titanium aluminum vanadium alloy. This square implant has an evenly ribbed structure on its upper and lower faces and an edge length of $25 \times 25$ $\mathrm{mm}$. The implant used in this study had a maximum height of $10 \mathrm{~mm}$ in the anterior segment with a dorsal inclination of 7 degrees.
The cylindrically shaped intervertebral disc dowel (IDD), manufactured by ESKA Implants $\mathrm{GmbH} \& \mathrm{Co}$, Luebeck, Germany, is made of a cobalt chrome molybdenum alloy and its surface has a three-dimensional tripodal webbed structure. The size of the IDD used in this study measured $35 \mathrm{~mm}$ in length and $15 \mathrm{~mm}$ in diameter. The German trade name of this implant is Bandscheibendübel ${ }^{\mathrm{TM}}$.

\subsection{Implantation}

Like in the human spine, the size of the vertebrae in the porcine spine increases in the craniocaudal direction, with the lower lumbar vertebrae extending to the maximum dimensions of $25 \mathrm{~mm}$ in height, 25 in width and 20 $\mathrm{mm}$ in depth. The dimensions of the 3 study spacers and the control were selected to be oversized compared to the intervertebral disc space. The two devices were implanted as stand-alone cages. We refrained from the use of dorsally implanted pedicle screws so as to avoid any potential summation effects on artifact scoring caused by additional materials.

A purely spinal model was chosen instead of a whole pig cadaver, since the size of the clinical field of view routinely focuses on the spine and cuts out any thoracic or abdominal organ structures. During dissection, the paravertebral muscles including the surrounding skin and the psoas muscles of the spine were retained. Special care was taken to ensure that the neurological structures of the spinal canal remained intact.

To determine the distance at which the spacers should be placed and to avoid artifact overlapping of spacers implanted in a single spine, we conducted a preliminary trial that involved embedding the cobalt-chrome IDD-the spacer with the highest magnetization-in a homogenous tissue mass and then performing MR imaging of the tissue-embedded spacer. The measurements showed that a width of $6.5 \mathrm{~cm}$ had to be maintained between spacers to avoid the artifact overlapping.

Accordingly, the lumbar and thoracic disc spaces were dissected to achieve a median positioning of the implants. The paravertebral muscles were left intact along with the 
skin and psoas muscles. Intervertebral discectomy was performed, and the three spacers and the control were implanted intervertebrally apart at a distance of $6.5 \mathrm{~cm}$. Maximum implantation depth was reached when the implant was aligned with the anterior vertebra face. After implantation was completed, additional tissue mass was padded around the spine to optimize contrast and image quality.

\section{MAGNETIC RESONANCE IMAGING}

MRI was performed with a 1.5T MRI (Magnetom Symphony, Siemens AG Medical Solutions, Erlangen, Germany). Table 1 presents the MRI data. The median sagittal MRI slice encompassing all relevant structures, implants and control was evaluated according to an interobserver-validated scoring system.

\subsection{Scoring System}

A 0-1-2 scoring system was established to rank the MRI scans. An evaluation unit was defined as 2 adjacent ver- tebrae encompassing the intervertebral disc space. 12 regions of interest (ROI) were demarcated (Figure 3). Every ROI could achieve a maximum score of 2 points. A total score of 24 points was equivalent to a score of $100 \%$. Two board-certified specialists (one radiologist $(\mathrm{GH})$ and one spinal surgeon (TE)) experienced in reading spinal MRI evaluated the scans independently of each other. The evaluators scored regions as $0=$ not distinguishable, $1=$ partly distinguishable and $2=$ completely distinguishable. The interobserver validation of the scoring system across all 15 sequences was tested for statistical significance using a $t$ test with a significance level of $P>0.05$ (Table 2).

\section{RESULTS}

Table 3 presents the total points scored for each implant in each of the 15 sequences. Figures 4 I, II, III and IV depict the artifact range in a selection of 4 MRI sequences.

Table 1. MRI sequence data.

\begin{tabular}{lcccccccc}
\hline \multicolumn{1}{c}{ Sequences } & FA & TR & TE & ST & BW & FOV & Number of slices & Matrix \\
\hline T1 FLASH 2D & 70 & 181 & 4.8 & 5.5 & 260 & 500 & 19 & $256 \times 256$ \\
T1 FLASH 2D FS & 70 & 275 & 4.76 & 5.5 & 260 & 500 & 19 & $256 \times 256$ \\
T2 MEDIC 2D FS & 40 & 2660 & 27 & 3.0 & 70 & 500 & 40 & $256 \times 256$ \\
T1 FLASH 3D & 60 & 60 & 11 & 3.0 & 70 & 500 & 40 & $256 \times 256$ \\
T2 DESS 3D & 25 & 23.68 & 6.63 & 1.5 & 130 & 500 & 64 & $256 \times 256$ \\
TOF FISP 3D & 25 & 36 & 4.59 & 3.0 & 130 & 500 & 32 & $384 \times 384$ \\
T2 CISS 3D & 70 & 10.16 & 5.08 & 3.0 & 130 & 500 & 64 & $256 \times 256$ \\
T1 TSE & 150 & 2260 & 14 & 3.0 & 150 & 500 & 40 & $512 \times 512$ \\
T1 TSE var & 150 & 600 & 14 & 3.0 & 150 & 500 & 40 & $512 \times 512$ \\
T1 SE & 90 & 1270 & 14 & 3.0 & 90 & 500 & 40 & $512 \times 512$ \\
T1 SE var & 90 & 600 & 14 & 3.0 & 90 & 500 & 40 & $512 \times 512$ \\
T1 SE FS var & 90 & 684 & 14 & 3.0 & 90 & 500 & 40 & $512 \times 512$ \\
T2 TSE/PD & 150 & 6110 & 14 & 3.0 & 130 & 500 & 40 & $256 \times 256$ \\
T2 TSE/PD FS & 150 & 6760 & 14 & 3.0 & 130 & 500 & 40 & $256 \times 256$ \\
STIR & 180 & 10000 & 38 & 3.0 & 130 & 500 & 40 & $256 \times 256$ \\
\hline
\end{tabular}

Key: FLASH=Fast Low Angle Shot, MEDIC=Multi Echo Data Image Combination, DESS=Dual Echo Steady State, FS=Fat Saturated, FISP $=$ Fast Imaging with Steady Precession, CISS=Constructive Interference in Steady State, SE=Spin Echo, $\mathrm{TSE}=$ Turbo Spin Echo, $\mathrm{PD}=$ Proton density, STIR=Short Tau Inversion Recovery, TOF=Time of Flight, TR=Time of Repetition, $\mathrm{TE}=$ Time of Echo, FA=Flip Angle, ST=Slab Thickness, BW=Band Width, FOV=Field of View, var=varied.

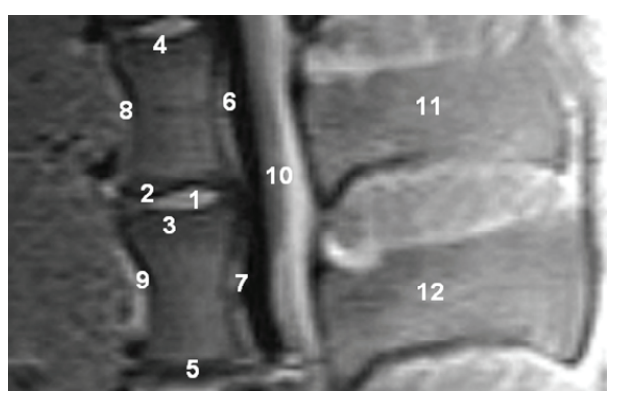

Figure 3. MRI evaluation unit with ROI.
Table 2. Interobserver validation across all 15 sequences.

\begin{tabular}{cccccc}
\hline & \multicolumn{2}{c}{ Mean score value } & \multicolumn{2}{c}{ Standard deviation } & \\
\cline { 2 - 5 } & $\begin{array}{c}\text { Spinal } \\
\text { surgeon }\end{array}$ & Radiologist & $\begin{array}{c}\text { Spinal } \\
\text { surgeon }\end{array}$ & Radiologist & \\
\hline IBS & 12.87 & 12.8 & \pm 1.25 & \pm 1.32 & $\mathrm{P}=0.58$ \\
IDD & 8.33 & 8.33 & \pm 3.02 & \pm 2.85 & $\mathrm{P}=1.0$ \\
Control & 20.13 & 20.4 & \pm 2.67 & \pm 2.79 & $\mathrm{P}=0.1$ \\
\hline
\end{tabular}


Table 3. Total scores.

\begin{tabular}{|c|c|c|c|c|c|c|}
\hline \multirow[t]{2}{*}{ MRI Sequence } & \multicolumn{2}{|c|}{ IBS } & \multicolumn{2}{|c|}{ IDD } & \multicolumn{2}{|c|}{ Control } \\
\hline & 1 & 2 & 1 & 2 & 1 & 2 \\
\hline $\begin{array}{l}\text { T1 FLASH 2D } \\
\text { TR: } 181 \quad \text { TE:4 }\end{array}$ & 13 & 13 & 9 & 8 & 18 & 18 \\
\hline $\begin{array}{l}\text { T1 FLASH 2D FS } \\
\text { TR: } 275 \text { TE: } 4\end{array}$ & 13 & 13 & 10 & 10 & 19 & 19 \\
\hline $\begin{array}{l}\text { T2 MEDIC 2D FS } \\
\text { TR:2660 TE:27 }\end{array}$ & 11 & 11 & 4 & 4 & 18 & 18 \\
\hline $\begin{array}{l}\text { T1 FLASH 3D FS } \\
\text { TR:60 TE: } 11\end{array}$ & 10 & 10 & 5 & 6 & 15 & 15 \\
\hline $\begin{array}{l}\text { T2 DESS 3D } \\
\text { TR:23 TE: } 6\end{array}$ & 13 & 12 & 6 & 6 & 21 & 21 \\
\hline $\begin{array}{l}\text { T2 CISS 3D } \\
\text { TR: } 10 \quad \text { TE: } 5\end{array}$ & 13 & 12 & 7 & 7 & 22 & 23 \\
\hline $\begin{array}{l}\text { T1 SE } \\
\text { TR:1270 TE:14 }\end{array}$ & 13 & 14 & 12 & 12 & 22 & 23 \\
\hline $\begin{array}{l}T 1 T S E \\
\quad T R: 2260 \quad T E: 14\end{array}$ & 15 & 15 & 12 & 12 & 24 & 24 \\
\hline $\begin{array}{l}T 1 T S E \\
\text { TR:600 TE:14 }\end{array}$ & 15 & 15 & 12 & 12 & 24 & 24 \\
\hline $\begin{array}{l}\text { T1 SE } \\
\text { TR:600 TE: } 14\end{array}$ & 13 & 13 & 12 & 12 & 23 & 23 \\
\hline $\begin{array}{l}\text { T1 SE FS } \\
\text { TR:684 TE: } 14\end{array}$ & 13 & 13 & 8 & 7 & 22 & 23 \\
\hline $\begin{array}{l}\text { PD+ T2 TSE } \\
\text { TR:6110 TE: } 14\end{array}$ & 13 & 13 & 8 & 8 & 19 & 20 \\
\hline $\begin{array}{cc}\text { PD+ T2 TSE } & \text { FS } \\
\text { TR:6760 TE: } 14\end{array}$ & 13 & 13 & 7 & 7 & 17 & 18 \\
\hline $\begin{array}{l}\text { STIR } \\
\quad \text { TR:10000 TE:38 }\end{array}$ & 13 & 13 & 10 & 10 & 19 & 19 \\
\hline $\begin{array}{l}\text { TOF FISP 3D } \\
\text { TR:36 TE:4 }\end{array}$ & 12 & 12 & 3 & 4 & 19 & 18 \\
\hline
\end{tabular}

Key: 1) spinal surgeon; 2) radiologist.
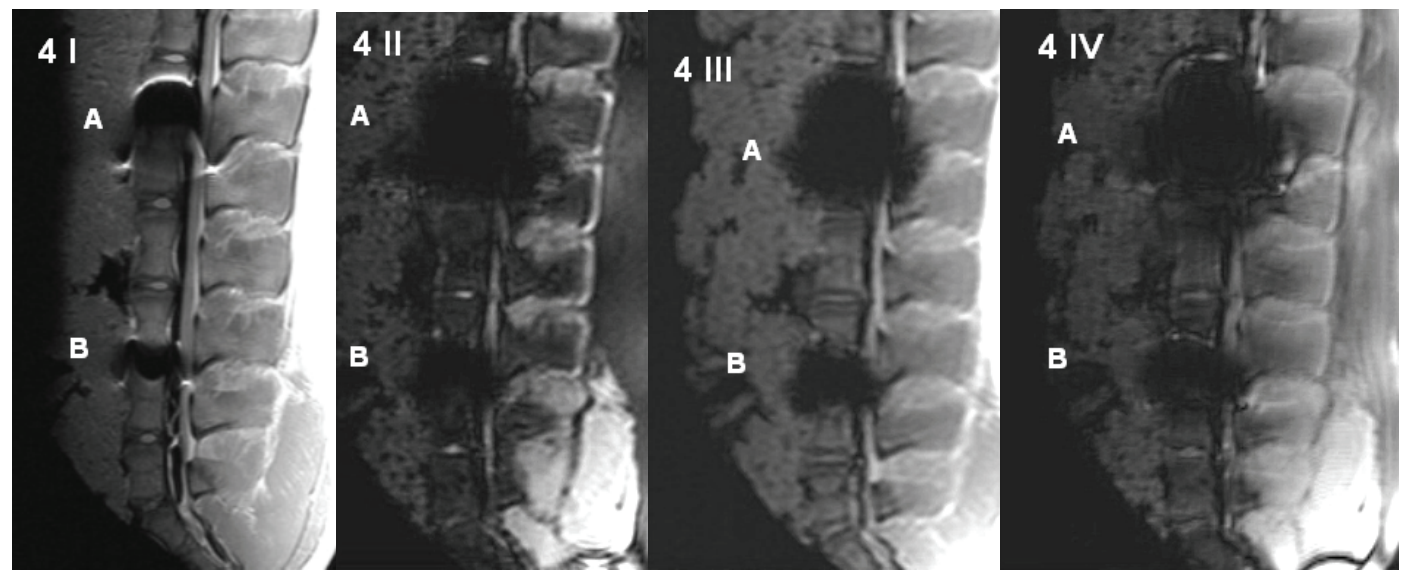

Figure 4. I-IV: Artifact range of the different MRI sequences [I: T1 TSE (TR: 2260, TE: 14); II: T2 DESS 3D (TR: 23, TE: 6); III: T1 FLASH 2D (TR: 181, TE: 4); IV: TOF FISP 3D (TR: 36, TE: 4)].

The results showed that the T1-TSE sequences produced the best imaging scores for all implants. In these sequences, the human cortical bone control achieved the maximum possible score of $100 \%$, i.e. was completely distinguishable (Figure 5, Table 3). Therefore, we used these two sequences as a basis for following comparison 
of the imaging quality of the study implants.

\subsection{IBS}

In the T1-TSE sequences, the titanium IBS implant achieved an imaging score of $62.5 \%$ compared to the control (Figure 4(IA), Table 3). The susceptibility artifact border was clearly distinguishable from its surroundings. As a result of artifact extension, the implant-bone contact area was not distinguishable. The image quality was not sufficient to determine exact implant position.

\subsection{IDD}

In the T1-TSE sequences, the IDD implant achieved an imaging score of $50 \%$ compared to the control (Figure 4 (IB), Table 3). As a result of artifacting, the implant-bone contact area was not distinguishable. The distant vertebral end plates were completely distinguishable; the anterior edge of the lower vertebra and the spinous process were partly distinguishable. As with the titanium IBS implant, the image quality was not sufficient to determine exact implant position in relation to the spinal canal.

\subsection{Interobserver Validation}

The results of the interobserver validation are listed in Table 3. There was no statistical significance between the evaluators with respect to t-test correlations $(P>0.05)$.

\section{DISCUSSIONS}

The disadvantages associated with bone grafting alone has led to the development of intervertebral spacers to enhance anterior spinal fusion $[5,6,7]$. The use of intervertebral spacers of different designs and materials has thus become increasingly widespread in clinical routine because they offering immediate load transmission with direct primary stability. Post-fusion MRI scans are used for further diagnostics to demonstrate any progressive degenerative changes, infections, fractures and/or tumors. However, implant-related susceptibility artifacts can negatively impact the complex post-fusion evaluation

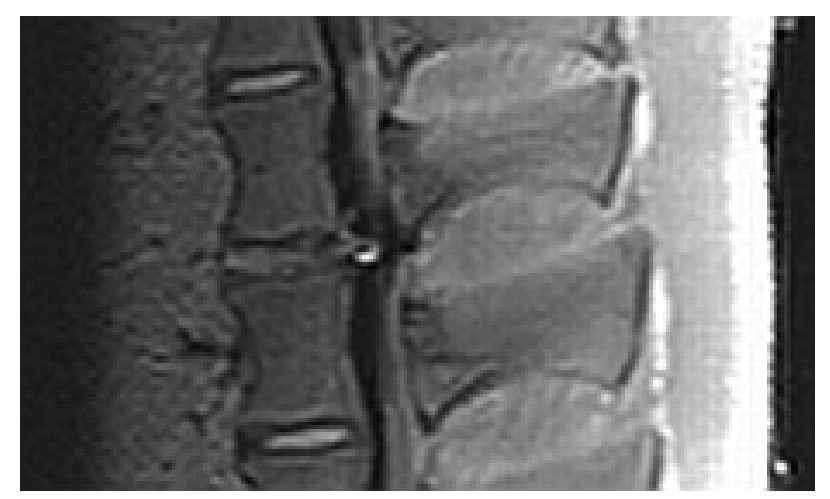

Figure 5. MRI slice human cortical bone (T1 TSE TR:2260 TE:14). of MRI scans. Depending on the spacer material, a local magnetic field gradient of varying susceptibility results in the area between structures. In these border areas, the respective spins gyrate with different frequencies and cause image distortions and susceptibility artifacts $[8,9,10]$.

Our image quality scoring system gave special consideration to the following material-related implant characteristics:

1) Distinguishability of implant shape and position;

2) Distinguishability of implant from anatomically neighboring structures;

3) The extent of image distortions and susceptibility artifacts.

Optimum MRI visualization of the different intervertebral spacers depends on the aim of diagnosis. MRI diagnostics are insofar subject to different requirements depending on the various postoperative pathologies in relation to the implant situation. Optimum MRI image distinguishability of the intervertebral spacers and the equivalent control was achieved using T1 TSE sequences. The imaging quality of the human cortical bone used as a control scored $100 \%$ according to the study scoring system and was therefore used as a basis to rank the intervertebral spacers examined. The scores were stated as a percentage compared to the control. Our interobservervalidated scoring system allowed us to create a unique implant-related ranking of MRI scan quality in reference to a control that was independent of artifact dimensions.

The MRI imaging behavior of metallic spinal implants is well documented in the literature $[9,10,11,12,13,14,15]$. However, the aims of the published studies differed in that most focused on determining sequence-related artifact size.

According to our study results the implant position in relation to the spinal canal_was best visualized using T1 TSE sequences. In studies by Rudisch et al. [16] and Thomsen et al. [17], titanium materials showed a lower artifact range than cobalt chrome. Consistent with our results, the best MRI quality was achieved for both metallic spacers with T1 TSE sequences. The other MRI sequences produced no further advantages.

Studies on metallic artifacts in MRI of the anterior spine have been conducted by Vaccaro et al. [15] and Wang et al. [18]. In one cadaveric study, Vaccaro et al. [15] examined the MRI artifact rates of different metal particles introduced in predefined intervertebral drill holes and subsequently embedded in paraffin. Vacarro could not demonstrate any significant artifacts in T1- or T2 SE sequences, probably due to the fact that the particle density was lower than that produced by metal implants commonly used in clinical practice. The metallic artifacts appearing in the gradient echo sequences proved a connection between artifact size and nickel content of the alloys examined. An increasing nickel content reduced susceptibility artifacting. 
In another cadaveric artifact study, Wang et al. [18] described the MRI behavior of an intervertebral spacer made of titanium. Using T1 SE sequences, the implantrelated artifact rate of the titanium spacer was primarily limited to the implant's direct surroundings and anatomic neighboring structures were clearly distinguishability. In our study, when T1 TSE sequences were used to image both metallic spacers, neither implant shape nor implant position could be distinguished with certainty. In a phantom study by Rudisch et al. [16], the relevance of metallic artifacts and implant-related characteristics, such as implant material, shape and position, was demonstrated in addition to an impact by the selected MRI sequence. In spite of the use of optimum MRI sequences, variability in the amount of susceptibility artifacts must be accounted for when evaluating MRI scans of metallic spine implants.

\section{CONCLUSIONS}

The designs and materials of the intervertebral spacers currently used in anterior spine fusion cause susceptibility artifacts that can be rated by validated scoring systems. Of 15 sequences tested, T1 TSE sequences produced the best spacer imaging for both metallic implants tested. An interobserver-validated scoring system proved effective in ranking the relevance of spacer material on MRI imaging quality. Studies are ongoing to further develop MRI scoring systems and establish optimum imaging sequences for post-fusion diagnostics.

\section{REFERENCES}

[1] Van Goethem, J.W., Parizel, P.M. and Jinkins, J.R. (2002) Review article: MRI of the postoperative lumbar spine. Neuroradiology, 44, 723-39.

[2] Herold, T., Caro, W.C., Heers, G., Perlick, L., Grifka, J., Feuerbach, S., Nitz, W. and Lenhart, M. (2004) Influence of sequence type on the extent of the susceptibility artifact in MRI: A shoulder specimen study after suture anchor repair. Rofo, 176, 1296-301.

[3] Schenck, J.F. (1996) The role of magnetic susceptibility in magnetic resonance imaging: MRI magnetic compatibility of the first and second kinds. Med Phys, 23, 815-50.

[4] Malik, A.S., Boyko, O., Atkar, N. and Young, W.F. (2001) A comparative study of MR imaging profile of titanium pedicle screws. Acta Radiol, 42, 291-3.

[5] Goulet, J.A., Senunas, L.E., DeSilva, G.L. and Greenfield, M.L. (1997) Autogenous iliac crest bone graft: Complications and functional assessment. Clin Orthop, 339,
76-81.

[6] Summers, B.N. and Eisenstein, S.M. (1989) Donor site pain from the ilium: A complication of lumbar spine fusion. J Bone Joint Surg Br, 71, 677-80.

[7] Brantigan, J.W. and Steffee, A.D. (1993) A carbon fiber implant to aid interbody lumbar fusion: Two-year clinical results in the first 26 patients. Spine, 18, 2106-7.

[8] Fellner, C., Behr, M., Fellner, F., Held, P., Handel, G. and Feuerbach, S. (1997) Artifacts in MR imaging of the temporomandibular joint caused by dental alloys: A phantom model study at T1.5. Rofo, 166, 421-8.

[9] Fritzsche, S., Thull, R. and Haase, A. (1994) Reduction of artifacts in magnetic resonance images by using optimized materials for diagnostic devices and implants. Biomed Tech (Berl), 39, 42-6.

[10] Henk, C.B., Brodner, W., Grampp, S., Breitenseher, M., Thurnher, M., Mostbeck, G.H. and Imhof, H. (1999) The postoperative spine. Top Magn Reson Imaging, 10, 247-64.

[11] Rupp, R., Ebraheim, N.A., Savolaine, E.R. and Jackson, W.T. (1993) Magnetic resonance imaging evaluation of the spine with metal implants: General safety and superior imaging with titanium. Spine, 18, 379-85.

[12] Ortiz, O., Pait, T.G., McAllister, P. and Sauter, K. (1996) Postoperative magnetic resonance imaging with titanium implants of the thoracic and lumbar spine. Neurosurgery, 38, 741-5.

[13] Petersilge, C.A., Lewin, J.S., Duerk, J.L., Yoo, J.U. and Ghaneyem, A.J. (1996) Optimizing imaging parameters for MR evaluation of the spine with titanium pedicle screws. AJR Am J Roentenol, 166, 1213-8.

[14] Wang, J.C., Sandhu, H.S., Yu, W.D., Minchew, J.T. and Delamarter, R.B. (1997) MR parameters for imaging titanium spinal instrumentation. J Spinal Disord, 10, 27-32.

[15] Vaccaro, A.R., Chesnut, R.M., Scuderi, G., Healy, J.F., Massie, J.B. and Garfin, S.R. (1994) Metallic spinal artifacts in magnetic resonance imaging. Spine, 19, 1237-42.

[16] Rudisch, A., Kremser, C., Peer, S., Kathrein, A., Judmaier, W. and Daniaux, H. (1998) Metallic artifacts in magnetic resonance imaging of patients with spinal fusion: A comparison of implant materials and implant sequences. Spine, 23, 692-9.

[17] Thomsen, M., Schneider, U., Breusch, S.J., Hansmann, J. and Freund, M. (2001) Artifacts and ferromagnetism dependent on different metal alloys in magnetic resonance imaging: An experimental study. Orthopade, 30, 540-4.

[18] Wang, J.C., Yu, W.D., Sandhu, H.S., Tam, V. and Delamarter, R.B. (1998) A comparison of magnetic resonance and computed tomographic image quality after the implantation of tantalum and titanium spinal instrumentation. Spine, 23, 1684-8. 